\title{
optica
}

\section{Slot-mode optomechanical crystals: a versatile platform for multimode optomechanics: supplementary material}

\author{
Karen E. Grutter*, M arcelo I. Davanço, and Kartik Srinivasan \\ Center for Nanoscale Science and Technology, National Institute of Standards and Technology, Gaithersburg, MD 20899-6203 USA \\ *Corresponding authors: karen.grutter@nist.gov, kartik.srinivasan@nist.gov
}

Published 19 November 2015

\begin{abstract}
This document provides supplementary information for "Slot-mode optomechanical crystals: a versatile platform for multimode optomechanics," http://dx.doi.org/10.1364/optica.2.000994. It describes the proce-dure for determining the phase modulator $V_{\pi}$, presents data from an additional triple-nanobeam device, explores how higher-order optical slot modes couple to various mechanical modes, and discusses thermo-optic effects in these slot-mode devices.
\end{abstract}

http://dx.doi.org/10.1364/optica.2.000994.s001

\section{MEASURING PHASE MODULATOR $V_{\pi}$}

In Sec. 4 of the main text, we use the phase modulator calibration method $[1,2]$ to measure the optomechanical coupling $g_{0}$ of slot-mode optomechanical crystals. The $V_{\pi}$ of the electro-optic phase modulator must be accurately known in order to do this calibration.

To measure $V_{\pi}$, we send the $980 \mathrm{~nm}$ laser signal through the phase modulator, modulated at $3.5 \mathrm{GHz}$ by an RF signal generator, and into a scanning Fabry-Pérot interferometer. The detected signal traces out the carrier and phase-modulatorinduced sidebands in the optical signal, and we view them on an oscilloscope, as shown in Fig. S1a.

Changing the power applied by the RF signal generator to the phase modulator changes the magnitude of the carrier and sidebands. The RF power $P_{\mathrm{RF}}$ is related to the signal voltage $V_{\text {sig }}=\sqrt{2 Z P_{\mathrm{RF}}}$, where the phase modulator input impedance $Z=50 \Omega$. Knowing this, we can graph the peak magnitudes with respect to $V_{\text {sig }}$, as shown in Fig S1b. For a phase modulator, the carrier peak magnitude should follow the curve $A\left(J_{0}\left(\pi V_{\text {sig }} / V_{\pi}\right)\right)^{2}$, and the first sideband magnitude should follow the curve $A\left(J_{1}\left(\pi V_{\text {sig }} / V_{\pi}\right)\right)^{2}$, where $A$ scales the amplitude of the Bessel functions of the first kind $J_{0}$ and $J_{1}$. We fit data from the carrier and first sideband to these functions in Fig. S1b, and both fits result in $V_{\pi}=2.78 \pm 0.01 \mathrm{~V}$, where the uncertainty comes from the fit and is one standard deviation. This value corresponds well with the vendor-specified value for the phase modulator.
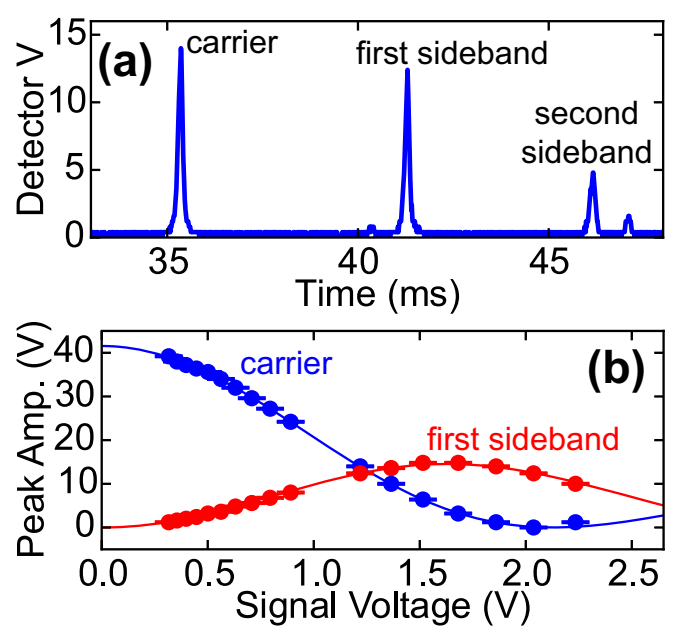

Fig. S1. (a) An example of the output of the scanning FabryPérot interferometer for a phase-modulated optical input signal, as read by an oscilloscope. Spectrum shows the carrier peak and the first and second sidebands. (b) Carrier (blue) and first sideband (red) peak heights with respect to RF signal voltage applied to the phase modulator. Points are the measured values, with error bars indicating the voltage resolution of the oscilloscope. Lines are the fits of the data. 

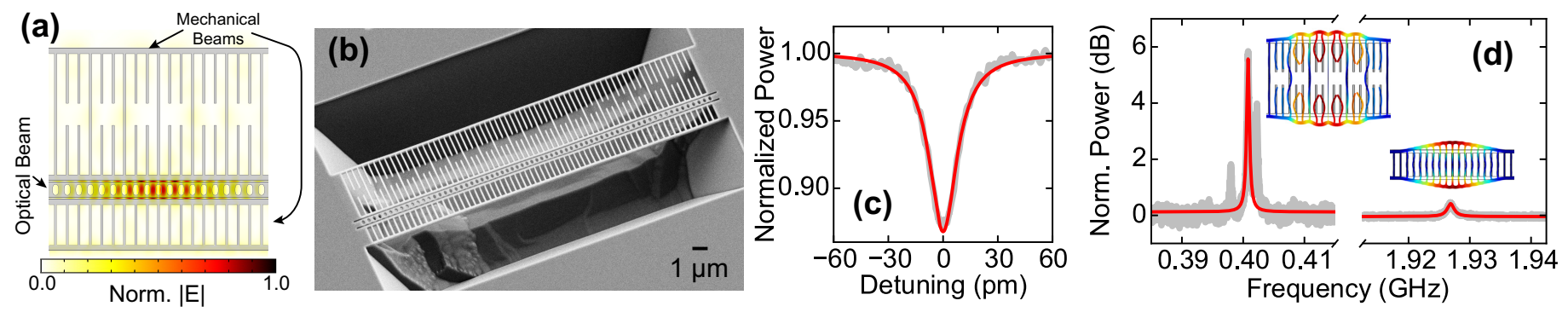

Fig. S2. (a) FEM simulation of the optical mode of an M-O-M device designed for coupling to $1.8 \mathrm{GHz}$ band (bottom beam) and $400 \mathrm{MHz}$ band (top beam) mechanical breathing modes. The optical mode is in both slots simultaneously. (b) SEM image of a fabricated M-O-M device. (c) Optical spectrum of M-O-M device. Measurement is in gray, and the Lorentzian fit is in red. Measured intrinsic $Q_{o}=(5.80 \pm 0.06) \times 10^{4}(\mathrm{~d})$ Both mechanical modes measured simultaneously, FTW input power $\approx 2 \mathrm{~mW}$. Data are in gray and Lorentzian fits are in red. At this optical input power, $400 \mathrm{MHz}$ mode has effective $Q_{m}=1030 \pm 20$, and 1.927 GHz mode has effective $Q_{m}=1450 \pm 20$, where uncertainty comes from $95 \%$ confidence interval of fit. (insets) FEM eigenmode simulations of corresponding mechanical breathing modes.

\section{ADDITIONAL M-O-M DEVICE}

In addition to the example M-O-M device in Sec. 6 of the main text of this work, we fabricated and characterized an M-O-M device in which a $400 \mathrm{MHz}$ band mechanical beam and a $1.8 \mathrm{GHz}$ band mechanical beam surround an optical beam, with $80 \mathrm{~nm}$ slots between the beams, shown in Fig. S2b. The resultant optical mode is concentrated in both slots simultaneously (Fig. S2a). We couple to the optical mode by hovering the FTW a few hundred nanometers above the optical beam. The measured intrinsic $Q_{o}$ was $(5.80 \pm 0.06) \times 10^{4}$ (Fig. S2c), and we simultaneously detect modulation of the transmitted optical signal from both the $400 \mathrm{MHz}$ band and $1.8 \mathrm{GHz}$ band mechanical modes (Fig. S2d). For the same input optical power, the detected $400 \mathrm{MHz}$ band mode has a larger amplitude than the $1.8 \mathrm{GHz}$ band mode primarily because a lower frequency mode has a larger thermal noise motional amplitude for the same temperature. As with the other devices in this work, the mechanical spectrum (Fig. S2d) includes other, less-well-coupled peaks that correspond to additional breathing-type mechanical modes from defects in the fabricated device [3] or harmonics of lowerfrequency flexural modes.

\section{COUPLING TO HIGHER-ORDER SLOT MODES}

These slot-mode optomechanical crystals confine multiple optical modes in the slot in addition to the fundamental mode for which they were designed. We observed some of these higher-order modes, as shown in Fig. S3a and b. Because they are distributed more widely along the slot, these modes couple less strongly to the highly-localized breathing mode and more strongly to other mechanical modes in the device, such as higher-order breathing-type mechanical modes arising from fabrication defects [3].

In the example of Fig. S3, a device with a designed, stresstuned slot width of $20 \mathrm{~nm}$ has an optical mode at $\approx 949 \mathrm{~nm}$ with an intrinsic optical $Q=(3.96 \pm 0.09) \times 10^{4}$ as well as an optical mode at $\approx 971 \mathrm{~nm}$ with an intrinsic optical $Q=$ $(3.57 \pm 0.08) \times 10^{4}$. When pumped at the $949 \mathrm{~nm}$ fundamental optical mode, it self-oscillates at the $\approx 3.52 \mathrm{GHz}$ mechanical breathing mode, and we begin to see sidebands due to mixing with the modulation from the low-frequency flexural beam modes, as described in Sec. 4 of the main text. However, even with $50 \%$ more optical power, the mechanical breathing mode does not self-oscillate when pumped at the $971 \mathrm{~nm}$ optical mode. In addition, the mechanical spectrum reveals another peak at $\approx 3.48 \mathrm{GHz}$ with about the same optomechanical coupling to the $971 \mathrm{~nm}$ mode as the $\approx 3.52 \mathrm{GHz}$ mode, suggesting that this higher-order optical mode is also coupling to some higher-order, less-well-confined mechanical mode.
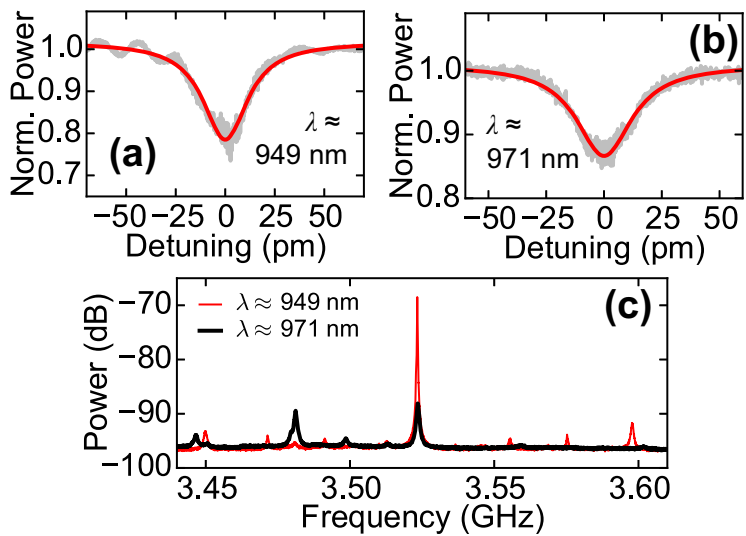

Fig. S3. (a) Fundamental optical slot mode at $\approx 949 \mathrm{~nm}$ with intrinsic $Q_{o}=(3.96 \pm 0.09) \times 10^{4}$. (b) Higher-order optical slot mode in the same device at $\approx 971 \mathrm{~nm}$ with intrinsic $Q_{o}=(3.57 \pm 0.08) \times 10^{4}$. (c) Mechanical spectra measured while coupled to the fundamental optical mode (red) and higher-order optical mode (black). The optical power input to the fiber taper waveguide was $\approx 2.2 \mathrm{~mW}$ at the $949 \mathrm{~nm}$ mode and $\approx 3.5 \mathrm{~mW}$ at the $971 \mathrm{~nm}$ mode. This power spectral density plot is referenced to a power of $1 \mathrm{~mW}=0 \mathrm{~dB}$.

\section{THERMO-OPTIC EFFECTS}

As the optical power coupled into a slot mode device increases, we see a shift in its resonant wavelength. This shift has been observed in other optical cavities, and is usually attributable to thermal expansion and index change [4].

In the case of the $\mathrm{Si}_{3} \mathrm{~N}_{4}$ slot-mode optomechanical crystals, we generally see a larger effective thermo-optic shift with respect to power than we see in single-nanobeam $\mathrm{Si}_{3} \mathrm{~N}_{4}$ optomechanical crystals [5], as shown in Fig. S4. Also, unlike in the single-nanobeam optomechanical crystals, there is some increase in the on-resonance transmission with respect to input 
power. We observe that the magnitude of these effects can vary greatly depending on device slot width and $Q_{o}$, which suggests that non-thermal effects may also be playing a role; we are continuing to investigate these matters.

Because of the extent of the optical-power-dependent resonance shift, red-detuned measurements will require some kind of active feedback to lock the laser to the cavity. Adding electrostatic functionality to these devices may facilitate this locking by enabling direct, on-chip tuning of the cavity wavelength.

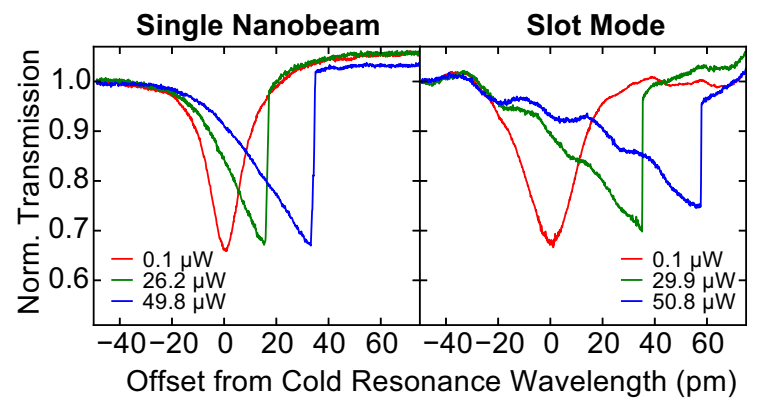

Fig. S4. A comparison of the effective thermo-optic shifts in a single-nanobeam $\mathrm{Si}_{3} \mathrm{~N}_{4}$ optomechanical crystal (left) and a $\mathrm{Si}_{3} \mathrm{~N}_{4}$ slot-mode optomechanical crystal (right). These devices have $Q_{o}$ s of $(7.9 \pm 0.1) \times 10^{4}$ and $(5.4 \pm 0.1) \times 10^{4}$, respectively. Optical spectra are traced out by a tunable laser scanning from shorter to longer wavelengths, with the dropped optical power $P_{d}=\left(1-T\left(\lambda_{0}\right)\right) P_{\text {in }}$ given in the legend.

\section{REFERENCES}

1. M. L. Gorodetsky, A. Schliesser, G. Anetsberger, S. Deleglise, and T. J. Kippenberg, "Determination of the vacuum optomechanical coupling rate using frequency noise calibration," Opt. Express 18, 23236 (2010).

2. K. C. Balram, M. Davanço, J. Y. Lim, J. D. Song, and K. Srinivasan, "Moving boundary and photoeleastic coupling in GaAs optomechanical resonators," Optica 1, 414-420 (2014).

3. M. Eichenfield, J. Chan, R. M. Camacho, K. J. Vahala, and O. Painter, "Optomechanical crystals," Nature 462, 78-82 (2009).

4. T. Carmon, L. Yang, and K. Vahala, "Dynamical thermal behavior and thermal self-stability of microcavities," Opt. Express 12, 4742-4750 (2004).

5. M. Davanço, S. Ates, Y. Liu, and K. Srinivasan, " $\mathrm{Si}_{3} \mathrm{~N}_{4}$ optomechanical crystals in the resolved-sideband regime," Appl. Phys. Lett. 104, 041101 (2014). 